\title{
THREE-DIMENSIONAL CIIARACTER OF SEMIMETALLIC InAs-GaSb SUPERLATTICES
}

\author{
J.C. Maan \\ Max-Planck Institut für Festkörperforschung, Hochfeld-Magnetlabor, 38042 Grenoble, France \\ Y. Guldner, J.P. Vieren, P. Voisin and M. Voos \\ Groupe de Physique des Solides de l'Ecole Normale Supérieure, ${ }^{*}$ Tour 23, 2 place Jussieu, 75005 Paris, France \\ and \\ L.L. Chang and L. Esaki
}

IBM Thomas J. Watson Research Center, P.O. Box 218, Yorktown Heights, NY 10598, U.S.A.

(Received 24 March 1981 by M. Balkanski)

\begin{abstract}
High-field, far-infrared magneto-absorption experiments are performed in semimetallic In As- $-\mathrm{GaSb}$ superlattices. The spectra exhibit extensive oscillations of cyclotron resonance and interband absorptions from valence to conduction subbands. Transitions at both the center and the boundary of the superlattice zone are observed, from which the width of the ground conduction subband is obtained, demonstrating dircctly its threedimensional character.
\end{abstract}

A CHARACTERISTIC FEATURE of the InAs-GaSb superlattice is the occurrence [1] of a semiconductorsemimetal transition when the InAs layer thickness becomes $\sim 100 \AA$. This transition arises from the peculiar band alignment of the basic materials; the bottom of the InAs conduction band being $150 \mathrm{meV}$ below the top of the GaSb valence band. Another inherent feature of this superlattice is associated with the extremely light electron mass of InAs. Its de Broglie wavelength is so long that, even for GaSb layer thickness in excess of $100 \AA$, there is considerable interaction between electrons in successive InAs layers which leads to conduction subbands with a substantial width in the range of tens of millivolts. This subband width manifests the quasi-three-dimensional character of a superlattice, as opposed to the situation arising from a simple quantum size effect.

The band structure of such a semimetallic superlattice is schematically shown in Fig. 1(a) along $k_{z}$, where $z$ is the direction perpendicular to the layers. The ground conduction subband $E_{1}$ is below the ground valence subband $H_{1}$. The conduction states are mainly localized in the InAs layers, and the valence states in the $\mathrm{GaSb}$ layers $[1,2]$. The width of $E_{1}$ is here $\Delta E_{1}$ but that of $H_{1}$ is essentially zero $[2,3]$. In the semimetallic samples, electrons transfer from $H_{1}$ to $E_{1}$, namely from $\mathrm{GaSb}$ to InAs, and the Fermi level $E_{F}$ is close to $H_{1}$.

\footnotetext{
* Laboratoire associé au Centre National de la Recherche Scientifique.
}

Under magnetic fields, both $E_{1}$ and $H_{1}$ exhibit a series of Landau levels, which move upward and downward, respectively, with an increase in the field. Clearly, each Landau level of $H_{1}$ gives rise to a single peak in the density of states. The $E_{1}$ subband, on the other hand, being flat at the superlattice zone center, $k_{z}=0$, and the zone boundary, $k_{z}= \pm \pi / d$, where $d$ is the superlattice periodicity, results in a doublet or two peaks in the density of states for each Landau level as shown in Fig. 1(b). If the selection rules for interband transitions are taken to be [4] $\Delta k_{z}-0$ and $\Delta N=0$, where $N$ is the Landau level index, two sets of transitions are thus possible at these two extremal points. Note that such interband transitions can occur because the electron and hole wavefunctions overlap.

We wish to present in this communication farinfrared magneto-absorption experiments in semimetallic In As-GaSb superlattices, similar to those reported earlier in establishing the negative energy gaps [4]. With the use of a high magnetic field and an optically pumped laser to provide a large number of infrared wavelengths, we have observed extensive transitions associated with both cyclotron resonance and interband absorptions with Landau indices covering the range of $N=1$ to 7 . These results not only substantiated the semimetallic property of the superlattice but provided accurate determinations of the subband encrgics of $E_{1}$ and $H_{1}$ at $k_{z}=0$. What is more significant is the observation of the transition between Landau levels at $k_{z}= \pm \pi / d$. The width of the $E_{1}$ subband has for the first time been 
Table 1. Thickness of the InAs $\left(\mathrm{d}_{1}\right)$ and $\mathrm{GaSb}\left(\mathrm{d}_{2}\right)$ layers for samples $\mathrm{S} 1$ and $\mathrm{S} 2$. Also given are the experimental and theoretical values of $\mathrm{E}_{1}, \mathrm{H}_{1}$ and $\Delta \mathrm{E}_{1}$

\begin{tabular}{|c|c|c|c|c|c|c|c|c|}
\hline \multirow[t]{2}{*}{ Samples } & \multirow[t]{2}{*}{$d_{1}(\AA)$} & \multirow[t]{2}{*}{$d_{2}(\AA)$} & \multicolumn{3}{|c|}{$\begin{array}{l}\text { Theory (LCAO) } \\
(\mathrm{meV})\end{array}$} & \multicolumn{3}{|c|}{$\begin{array}{l}\text { Experiment } \\
(\mathrm{meV})\end{array}$} \\
\hline & & & $E_{1}$ & $\Delta E_{1}$ & $H_{1}$ & $E_{1}$ & $\Delta E_{1}$ & $H_{1}$ \\
\hline S1 & 120 & 80 & 87.5 & 21 & 129.5 & $100 \pm 15$ & $23 \pm 1$ & $139 \pm 15$ \\
\hline S2 & 200 & 100 & 64.5 & 16 & 132.5 & $70 \pm 10$ & $19 \pm 1$ & $128 \pm 10$ \\
\hline
\end{tabular}

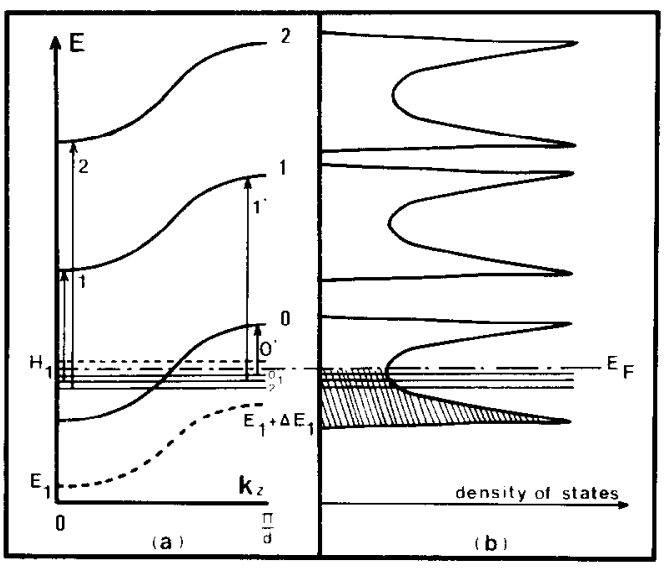

Fig. 1. (a) Schematic band structure of a semimetallic InAs-GaSb superlattice along $k_{z}$. The dashed curves are without magnetic field, the solid curves are the corresponding Landau levels under magnetic field, and $E_{F}$ is the Fermi level. Transitions noted 1,2 correspond to transitions at $k_{z}=0$ from $H_{1}$ Landau levels up to $E_{1}$ Landau levels with the same index, namely $N=1,2$. Those noted $0^{\prime}, 1^{\prime}$ correspond to similar transitions at $k_{z}-\pi / d$, with $N=0,1$. (b) Density of states assuciated with the $E_{1}$ and $H_{1}$ subbands under magnetic field. The hatched area corresponds to states occupied by electrons.

measured experimentally, which indicates directly that the electron system in a superlattice possesses a threedimensional character, deviating from strict twodimensionality.

The samples (S1 and S2) used here were grown [1] by molecular-beam epitaxy on (100) GaSb substrates, the total thickness of the superlattice being typically $\sim 2 \mu \mathrm{m}$. The thicknesses of the InAs and GaSb layers are given in Table 1 for both samples. Experimentally, the FIR transmission of these superlattices is studied at fixed photon energies as a function of the magnetic field $B$. The infrared radiation is propagating parallel to $B$ (Faraday configuration), which is applied perpendicular to the plane of the layers. The field was provided by a Bitter magnet and could be varied continuously from 0 to $20 \mathrm{~T}$. Several FIR wavelengths were generated using an optically pumped FIR laser with $\mathrm{CH}_{3} \mathrm{OH}$ as the active medium $(\lambda=1217,699,570,471,394,294,254,233$, $203,193,170,118,96$ and $70 \mu \mathrm{m})$. The transmission

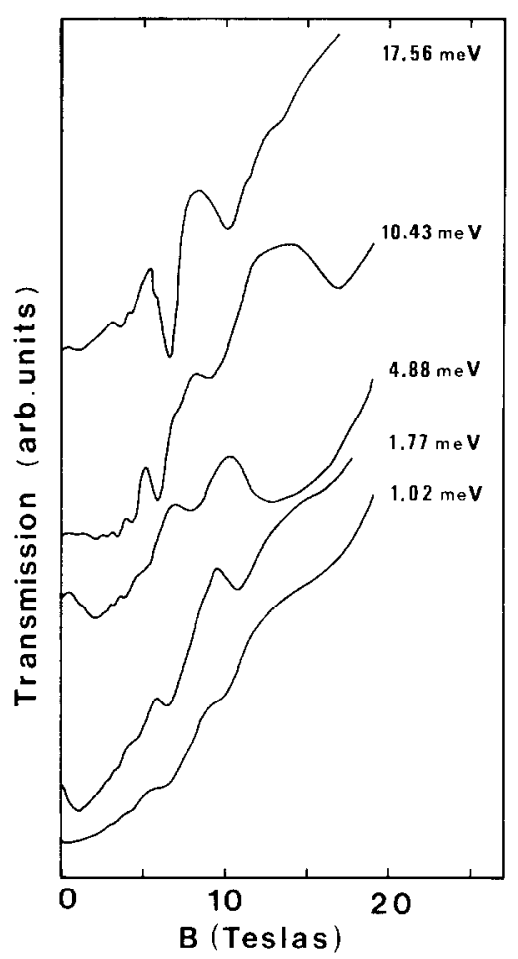

Fig. 2. Transmission signals vs. magnetic field observed at liquid helium temperature in sample S1 for different infrared photon energies.

signal was measured with a Ge bolometer cooled at liquid helium temperature, and normalized to the incident radiation intensity with a reference signal detected with a Golay cell to eliminate fluctuations of laser instabilities.

Typical transmission spectra obtained at liquid helium temperature are presented in Fig. 2 for sample $\mathrm{S} 1$, where it can be seen that, for each infrared wavelength, the transmission signal as a function of $B$ exhibits several minima. In Fig. 3 are presented the infrared energy positions of the transmission minima (or absorption maxima) vs. $B$. As shown below from theoretical analyses, the energies corresponding to the transmission minima depend almost linearly on $B$. Extrapolation to zero field results in $h \nu=0$ for the line marked CR in Fig. 3(a), an energy of $-39 \mathrm{meV}$ for the family of lines 

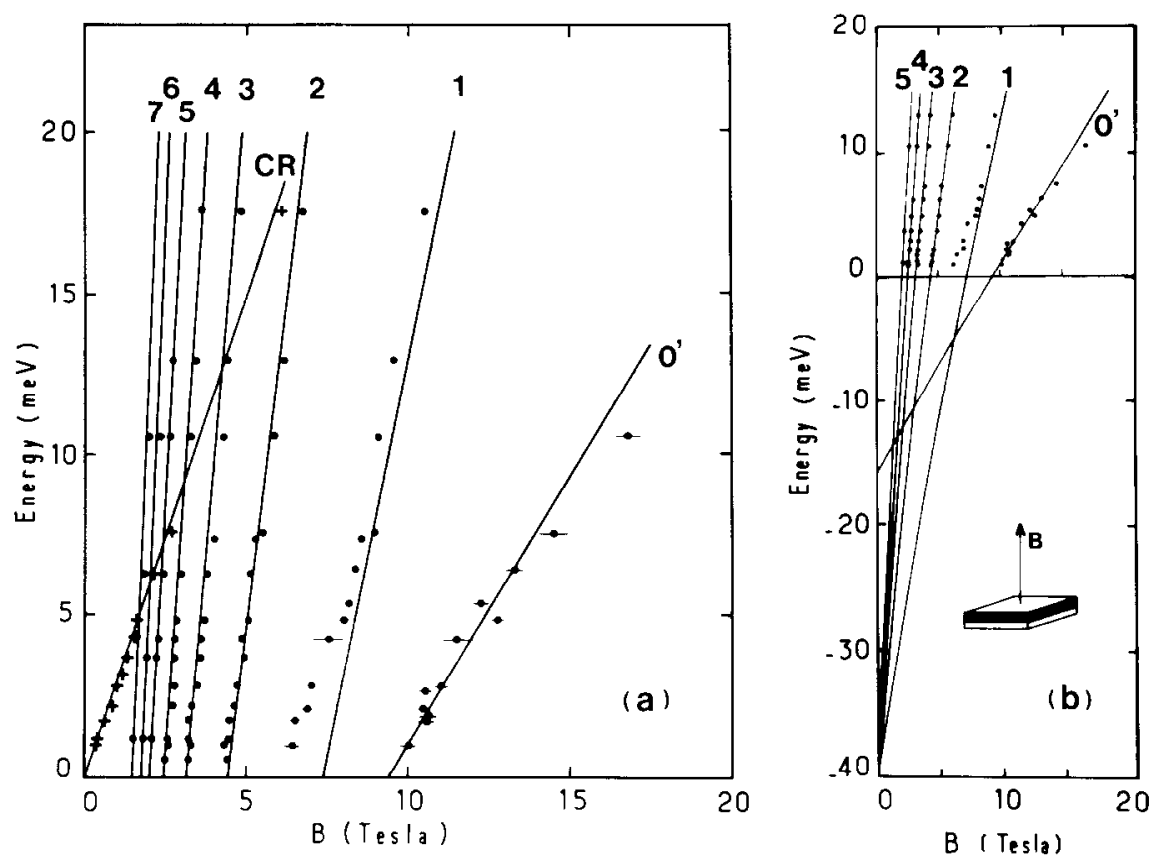

Fig. 3. Position of the transmission minima (see Fig. 2) as a function of the infrared photon energy and magnetic field $B$ (crosses and full dots). The solid lines correspond to theoretical fits using the model presented in the text. The inset shows schematically the geometry of the experiment.

designated 1, 2, 3, . in Figs. 3(a) and 3(b), and an energy of $-16 \mathrm{meV}$ for the single line which is denoted as $0^{\prime}$ in the same figures. The CR line is interpreted as being due to electron cyclotron resonance, i.e. to transitions from the last filled to the first empty Landau level of $E_{1}$. The curves which converge to the most negative energy are attributed to interband transitions from $H_{1}$ to $E_{1}$ Landau levels at $k_{z}=0$, such as those noted 1 and 2 in Fig. 1(a). The $0^{\prime}$ line is also attributed to similar interband transitions, but it occurs at $k_{z}=\pi / d$ as illustrated in Fig. 1(a). These interpretations in terms of both cyclotron resonance and interband transitions are consistent with those reported earlier [4]. The observations of additional transitions associated with the 1 and $0^{\prime}$ branches are made possible by the application of high fields in the present experiments.

Quantitatively the $E_{1}$ and $H_{1}$ Landau levels are calculated as were done previously $[4,5]$, taking into account the InAs conduction band non-parabolicity on the basis of the two-band $k . p$ model due to Kane [6]. For the $E_{1}$ subband one obtains for the $N$ th Landau level:

$E_{1, N}=-\frac{E_{G}}{2}+\left[\left(\frac{E_{G}}{2}\right)^{2}+E_{G} D_{N}\right]^{1 / 2}$

with $D_{N}=(N+1 / 2) \hbar_{\omega_{c}}+E_{1}\left(1+E_{1} / E_{G}\right)$ and $\omega_{c}=$ $e B / m_{e}^{*}$ where $E_{G}$ and $m_{e}^{*}$ are respectively the band gap and band edge mass of bulk InAs. For the $H_{1, N}$ Landau level, we take simply $H_{1, N}=H_{1}-(N+1 / 2) \hbar \omega_{v}$, with $\omega_{v}=e B / m_{h}^{*}$ where $m_{h}^{*}$ is the GaSb heavy hole effective mass. All the energies used here are measured from the bulk InAs conduction band edge.

For electron cyclotron resonance transitions, $h \nu=E_{1, N+1}-E_{1, N}$, where $N$ is such that $E_{1, N}$ is below, and $E_{1, N+1}$ above, the Fermi level $E_{F}$. Interband transitions occurring at $k_{z}=0$ from $H_{1}$ Landau levels up to $E_{1}$ Landau levels correspond to $h \nu=E_{1, N}-H_{1, N}$, taking also here $E_{F}=H_{1}$ and the same selection rules as in [4]. Fits of our experimental data to this theoretical model are shown in Fig. 3 for sample S1, with $m_{e}^{*}=0.023 m_{0}, E_{G}=410 \mathrm{meV}$ and $m_{h}^{*}=0.33 m_{0}$ [7]. The agreement between experiment and theory is quite good and, from these fits, we obtain the values of $E_{1}$ and $H_{1}$ at $B=0$ which are listed [8] in Table 1. Similar studies were done in sample $\mathbf{S} 2$, and the corresponding values of these parameters are also given in Table 1. We can, in addition, determine the superlattice electron cyclotron mass $m^{*}$ from the CR data, which is found to be [8] $0.0385 m_{0}$ and $0.0375 m_{0}$ in samples $S 1$ and $S 2$, respectively, with an uncertainty of $\pm 0.0015 \mathrm{~m}_{0}$.

Having established the values of $E_{1}$ and $H_{1}$, we can calculate the transition energies at $k_{z}=\pi / d$ from the same theoretical modcl, replacing $E_{1}$ by $E_{1}+\Delta E_{1}$ in equation (1). Figure 3 shows the results fitted to the data points from which the Landau index is identified, $N=0^{\prime}$, and the bandwidth is obtained, $\Delta E_{1}=23 \mathrm{meV}$. This value together with that obtained from sample $S 2$ is also listed in Table 1. To observe the transition at $k_{z}=\pi / d$ requires that the subband broadening from 
scattering be smaller than the subband width itself. This is borne out from the scattering time of $\sim 5 \times 10^{-13} \mathrm{sec}$ as estimated from the cyclotron resonance linewidth, which corresponds to a level broadening of $\sim 1.2 \mathrm{meV}$. That transitions associated with higher Landau indices at this zone boundary are not observed experimentally can be understood from considerations of wavefunction overlap between electrons and holes [9]. For a given energy, the $E_{z}$ component at $k_{z}=\pi / d$ is higher than that at $k_{z}=0$ by the amount of $\Delta E_{1}$. This brings the former closer to the valence band edge of $\mathrm{GaSb}$ as such its associated electron wavefunction is more localized in InAs. For a given magnetic field, transitions at $\pi / d$ are expected to bc weaker than at 0 , so that they can bc resolved only in regions where transitions at $k_{z}=0$ are not present. It is clear from Table 1 , where calculated values of $E_{1}, H_{1}$ and $\Delta E_{1}$ by the LCAO method are also included, that the experimental and theoretical results compare quite favorably in all cases.

The systematic deviation around $8 \mathrm{~T}$ of the data points from the calculated curve for the $N=1$ transition, as can be seen in Fig. 3, is not understood at present. This may be due to polaron effects, which have been observed for interband transitions [10]. The effects become important at magnetic field values when the energy separation between the $N=0$ and the $N=1$ Landau levels, for example, approaches the longitudinal optical phonon energy. That the LO-phonon energy of In As (and $\mathrm{GaSb}$ ) is [7] about $30 \mathrm{meV}$ is consistent with our data. Recent calculations [11] have shown that the magneto-optical anomalies arising from electronphonon interactions are considerably enhanced in superlattices.

To conclude, we have observed in semimetallic InAs- $\mathrm{GaSb}$ superlattices cyclotron resonance and interband transitions at $k_{z}=0$, from Landau levels of the ground valence subband $H_{1}$ up to those of the ground conduction subband $E_{1}$. We have obtained extensive experimental data, corroborating earlier results reported previously [4]. In addition, we have deter- mined the width $\Delta E_{1}$ of the ground conduction subband, demonstrating directly the quasi-three-dimensional character of these superlattices. Finally, we have obtained from these investigations values of $E_{1}, H_{1}$ and $\Delta E_{1}$ which compare favorably with those calculated by the LCAO method [2].

Acknowledgements - The authors acknowledge the valuable technical assistance of W. Schmid in the experimental part of this work. The E.N.S. group would like to thank G. Landwehr for his hospitality at the MaxPlanck Institüt in Grenoble where these experiments were done, and one of us (J.C.M.) for his interest in this work. The IBM group acknowledges partial sponsorship of the work under U.S. Army Research Office Contract.

\section{REFERENCES}

1. For a recent review see, for example, L.L. Chang \& L. Esaki, Surf. Sci. 98, 70 (1980).

2. G.A. Sai-Halasz, L. Esaki \& W.A. Harrison, Phys. Rev. B18, 2812 (1978).

3. Note that $H_{1}$ corresponds to heavy holes (see, for example, [1]).

4. Y. Guldner, J.P. Vieren, P. Voisin, M. Voos, L.L. Chang \& L. Esaki, Phys. Rev. Lett. 45, 1719 (1980).

5. H. Bluyssen, J.C. Maan, P. Wyder, L.L. Chang \& L. Esaki, Solid State Commun. 31, 75 (1979).

6. E.O. Kane, J. Phys. Chem. Solids 1, 249 (1957).

7. The InAs and GaSb parameters can be found in Handbook of Electronic Materials (Edited by M. Neuberger), Vol. 2. Plenum, New York (1971).

8. The values of $E_{1}, H_{1}$ and $m^{*}$ obtained here are somewhat different from those reported in [4]. This is due to different values of the InAs band gap used in the theoretical fits. Indeed, the fits shown in [4] were done with a gap of $420 \mathrm{meV}$, not $410 \mathrm{meV}$ as erroneously written in the text.

9. G.A. Sai-Halasz, L.L. Chang, J.M. Welter, C.A. Chang \& L. Esaki, Solid State Commun. 26, 935 (1978).

10. E.J. Johnson \& D.M. Larsen, Phys. Rev. Lett. 16, 655 (1966).

11. S. Das Sarma \& A. Madhukar, Phys. Rev. B22, 2823 (1980). 